\title{
Development and Validation of a Nomogram for Predicting Postoperative Pulmonary Infection in Esophageal Cancer
}

Shuang LI

Shandong University

Jingwen Su

Shandong University

Qiyu Sui

Shandong University

Gongchao Wang ( $\square$ wanggongchao@126.com )

Shandong University

\section{Research Article}

Keywords: Esophageal Cancer, Post-operative Pulmonary Infection, Nomogram

Posted Date: April 7th, 2021

DOI: https://doi.org/10.21203/rs.3.rs-391909/v1

License: (c) (i) This work is licensed under a Creative Commons Attribution 4.0 International License. Read Full License 


\title{
Development and Validation of a Nomogram for Predicting Postoperative Pulmonary Infection in Esophageal Cancer
}

Shuang Li, Jingwen Su, Qiyu Sui, Gongchao Wang*

School of Nursing, Shandong University, Jinan 250012, China.

Corresponding Author: Gongchao Wang, Tel.:+86 151-6886-3772, Email:wanggongchao@126.com

\begin{abstract}
Objective: The main aim of this study is to construct and validate a nomogram for estimating the risk of $\mathrm{POI}$ by investigating how perioperative features contribute to POI.
\end{abstract}

Material and Methods: This cohort study enrolled 637 patients with esophageal cancer. Perioperative information on participants were collected to develop and validate a nomogram for predicting postoperative pulmonary infection in esophageal cancer. Predictive accuracy, discriminatory capability and clinical usefulness were evaluated by calibration curves, concordance index (C-index) and decision curve analysis (DCA).

Results: Multivariable logistic regression analysis indicated that length of stay, albumin, intraoperative bleeding, and perioperative blood transfusion were independent predictors of $\mathrm{POI}$. The nomogram for assessing individual risk of $\mathrm{POI}$ indicated good predictive accuracy in the primary cohort (C-index, 0.802) and validation cohort (C-index, 0.763). Good consistency between predicted risk and observed actual risk was presented as the calibration curve. The nomogram for estimating $\mathrm{POI}$ of esophageal cancer had superior net benefit with a wide range of threshold probabilities (4-81\%).

Conclusions: The present study provided a nomogram developed with perioperative features to assess the individual probability of infection may conducive to strengthen awareness of infection control and provide appropriate resource to manage patients at high-risk following esophagectomy.

Keywords: Esophageal Cancer; Post-operative Pulmonary Infection; Nomogram

\section{Introduction}

Esophageal cancer (EC) was diagnosed with 572,034 new cases and results in 508,585 deaths around the world in 2018 [1]. The past few decades have seen rapid increase in the incidence of esophageal cancer [2]. Although multimodal therapy composed of surgical treatment, radiotherapy, and chemotherapy, prognosis outcomes remain poor for EC, with only $15 \%-25 \%$ of patients surviving beyond 5 years [3]. For patients with esophageal cancer, esophagectomy remains the primary option for esophageal cancer patients because of removing the tumors of the esophagus and improving symptoms. Despite the incidence and mortality of esophageal cancer decline resulting from medical advances and perioperative management, postoperative pulmonary complications, especially postoperative pulmonary infection (POI), which is a principle problem associated with patient's prognosis and outcomes [4]. Several studies reported that $\mathrm{POI}$ occurred in almost $16 \%-40 \%$ of EC patients and has been identified to be a factor for perioperative death and long-term survival [5-7]. It needs to, therefore, distinct and identify those patients at the greatest risk of $\mathrm{POI}$, and promote early intervention to reduce its incidence or improve postoperative prognosis outcomes. A study observed that the increasing POI rate connected with several risk factors such as age, smoking, preoperative comorbidity, 
lower hemoglobin, higher creatinine, postoperative dysphagia[8-10]. However, the risk factors of POI following esophageal cancer resection are inconclusive, showing the differences in institutions and healthcare delivery facilities.

Construct a mathematical model to predict $\mathrm{POI}$ may be a solution to the issue. A nomogram is a graphical depiction which presents a regression model on a friendly manner and simplify risk assessment, offering healthcare practitioners a user-friendly interface to map probability of an event to individual patients and enhancing clinical decision-making of both medical personnel and patients $[11,12]$. Such a device would strengthen the validity and objectivity of risk assessment. Therefore, this study seeks to develop and validate a nomogram for predicting $\mathrm{POI}$ with perioperative information.

\section{Materials and Methods}

\section{Study Population}

This retrospective cohort study involved adult patients with a newly diagnosed EC from January 1,2010, to December 1, 2011 at the Shandong Provincial Hospital. Study samples and treatment data were retrieved from the database of respective surgical departments. Patients will be included in this study if they meet the following requirements:(1) no less than 18-year age, (2) pathological section diagnosed as malignant esophageal cancer (3) underwent curative esophagectomy. On the other hand, if EC patients who died within 24 hours after surgery or lack complete case records were excluded.

\section{Study Outcomes and Data collection}

The primary endpoint POI, definition referred to Centers for Disease Control and Prevention and National Healthcare Safety Network surveillance definition [13]. Pathological staging was performed according to the American Joint Committee on Cancer (AJCC) Staging Handbook (7th edition) [14].The following data were collected: gender, age, length of stay, body mass index, smoking, drinking, hypertension, diabetes mellitus, coronary heart disease, chronic obstructive pulmonary disease, pulmonary tuberculosis, tumor type, primary tumor site, AJCC pathological stage, AJCC clinical stage, chemoradiotherapy, lymph node metastasis, forced vital capacity, forced vital capacity percentage predicted, forced expiratory volume in one second, forced expiratory volume in one second percentage predicted, Albumin, hemoglobin, pattern of anastomosis, surgery time, intraoperative bleeding, perioperative blood transfusion, American society of anesthesiologists score, postoperative pulmonary infection.

\section{Statistical analysis.}

Continuous variables were reported as mean with standard deviation (SD) and categorical variables as frequency with percentage. The Least absolute shrinkage and selection operator (LASSO) regression model was performed to tackle the collinearity of candidate variables to select the optimal predictive variables [15]. Multivariable logistic regression analysis was generated using selected predictors from LASSO analysis. The features were presented as odds ratio $(O R)$ and $95 \%$ confidence interval $(\mathrm{Cl})$. A two-tailed $P$ value $<0.05$ was considered statistical significance. Model discrimination was assessed by concordance index ( $\mathrm{C}$-index) and calibration was evaluated by calibration curve. Decision curve analysis (DCA) was adopted to determine the clinical usefulness and net benefit of the nomogram [16]. Externally validation was generated to confirm the stability of the nomogram in the validation cohort using 1000 
bootstrap resamples and calculating a relatively corrected C-index. Statistical analyses were carried out using SPSS, version 25.0 and R Studio, version 4.0.2.

\section{Ethical approval.}

The data used in this study was approved by the Institutional Review Board of Shandong University, and was exempt from the requirement for individual patient consent because contained no personal identifiers. The study complied with the principles of Declaration of Helsinki.

\section{Results}

\section{Population Characteristics}

Table 1 shows the clinical characteristics of the study population. A total of $637 \mathrm{EC}$ patients were enrolled in this study, separated by training cohort (from January 1, 2010, to July 31, 2011) and validation cohort (from August 1, 2011, to December 31, 2011). Of 446 patients (mean [SD] age, 59.77 [8.3] years; 349 men [78.3\%]) in training cohort, 95 patients (21.3\%) were diagnosed POI, while 191 patients (mean [SD] age, 58.47[8.7] years; 170 men [89.0\%]) composed of validation cohort, 36 patients (18.8\%) experienced POI.

\section{Selected predictors}

Of 28 features, 4 potential predictors were finally selected on the basis of LASSO regression analysis (Fig.1). The optimal predictors included length of stay, albumin, intraoperative bleeding, perioperative blood transfusion.

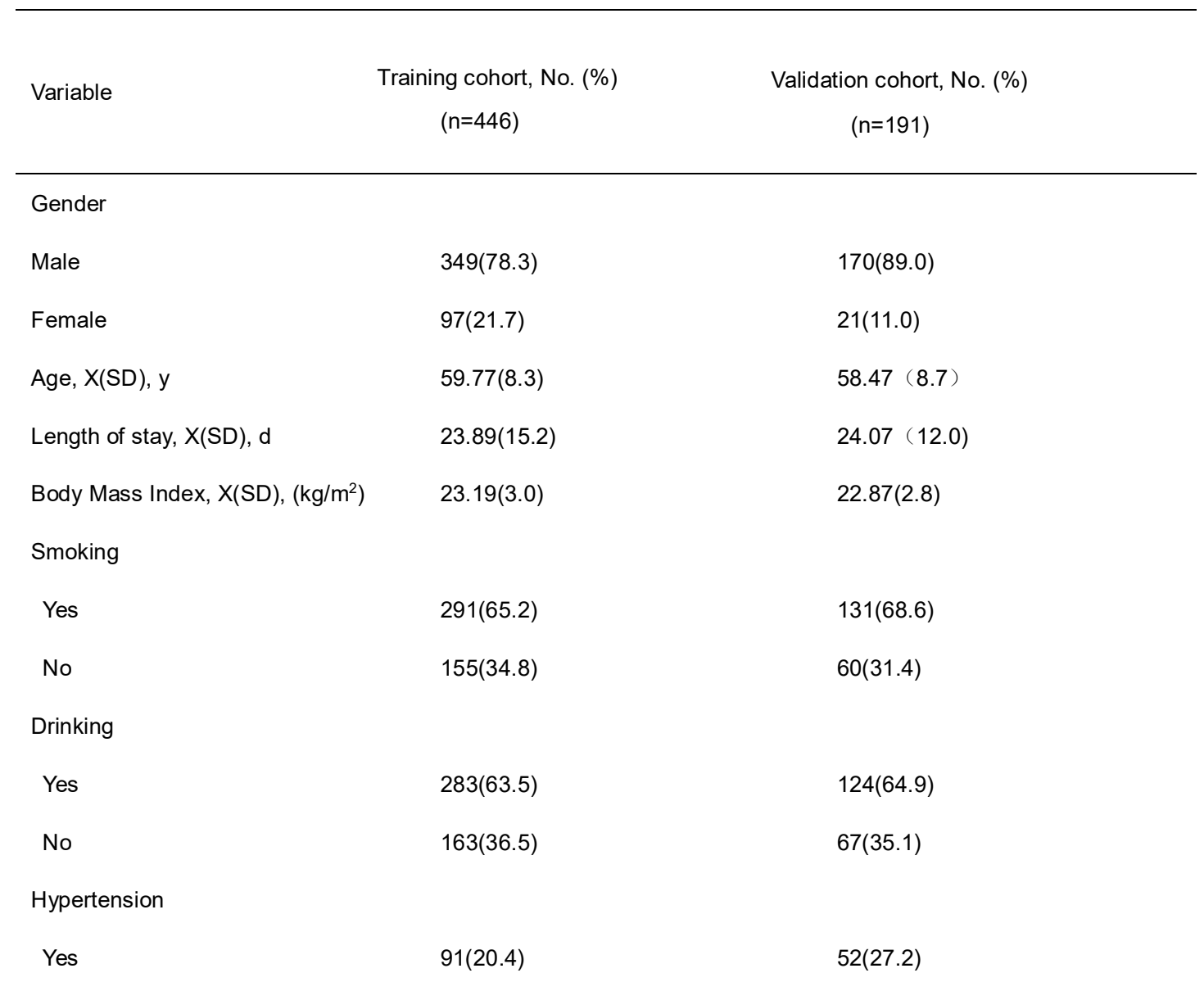


No

$355(79.6)$

Diabetes mellitus

Yes

No

Coronary heart disease

Yes

No

COPD

Yes

No

pulmonary tuberculosis

Yes

No

Tumor type

Squamous cell carcinoma

Adenocarcinoma

Other

Primary tumor site

Upper

Middle

Lower

Others

AJCC pathological stage

1

2

3

4

\section{AJCC clinical stage}

1

2

3

4

Chemoradiotherapy
38(8.5)

16(8.4)

408(91.5)

175(91.6)

22(4.9)

11(5.8)

424(95.1)

180(94.2)

$3(0.7)$

O(0)

443(99.3)

191(100)

27(6.1)

13(93.2)

419(93.9)

178(6.8)

419(93.9)

181(94.8)

14(3.1)

6(3.1)

13(3)

4(2.1)

26(5.8)

9(4.7)

242(54.3)

108(56.6)

137(30.7)

55(28.8)

41(9.2)

19(9.9)

24(5.4)

11(5.8)

$339(76)$

136(71.2)

82(18.4)

44(23)

$1(0.2)$

$0(0)$

$50(11.2)$

22(11.5)

225(50.4)

99(51.9)

171(38.4)

69(36.1)

$0(0)$

1(0.5) 


$$
\text { Yes }
$$$$
\text { No }
$$

Lymph node metastasis

$$
\text { Yes }
$$$$
\text { No }
$$

FVC, X(SD), L

FVC\%pred, X(SD), \%

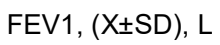

FEV1\%pred, X(SD), \%

Albumin (g/L), (X士SD)

Hemoglobin (g/L), X(SD)

Pattern of anastomosis

1

2

3

4

Surgery time, $\mathrm{h}$

$$
\leq 3
$$

$>3$

Intraoperative bleeding,X(SD), ml

Perioperative blood transfusion

Yes

No

ASA score

$$
1
$$

\begin{tabular}{|c|c|c|}
\hline Yes & $95(21.3 \%)$ & $36(18.8 \%)$ \\
\hline No & 351 (78.7\%) & $155(81.2 \%)$ \\
\hline
\end{tabular}$$
2
$$$$
3
$$

Postoperative pulmonary infection

Table 1. Characteristics of the study population. COPD: chronic obstructive pulmonary disease; AJCC: American Joint Committee on Cancer; FVC: forced vital capacity; FVC\% pred: forced vital capacity percentage predicted; FEV1: forced expiratory volume in one second; FEV1\% pred: forced expiratory volume in one second percentage predicted; ASA: American society of anesthesiologists.
$0(0)$

191(100)

87(45.5)

104(54.5)

$5.18(27.1)$

$90.93(15.4)$

$2.77(0.7)$

97.39(18.4)

$32.40(3.8)$

120.69(17.8)

$82(42.9)$

49(25.7)

13(6.8)

$47(24.6)$

62(32.5)

129(67.5)

213.98(159.8)

60(31.4)

131(68.6)

27(14.1)

155(81.2)

9(4.7)

$34(7.6)$

$36(18.8 \%)$

$155(81.2 \%)$ 
A

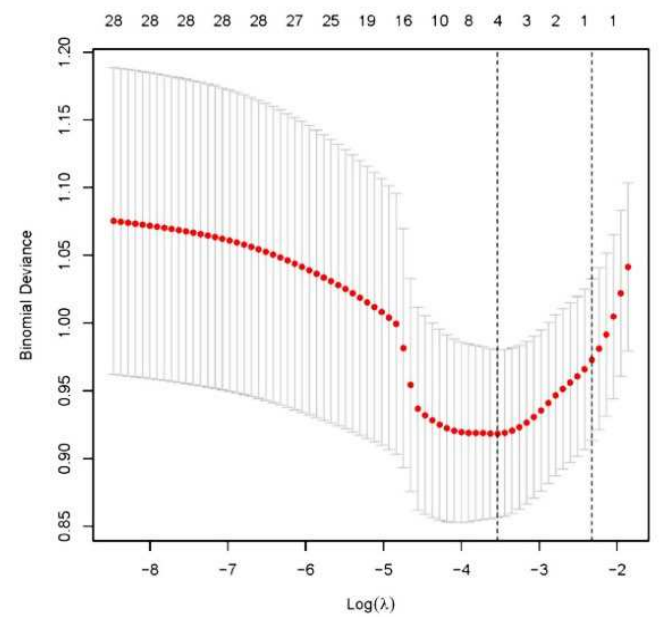

B

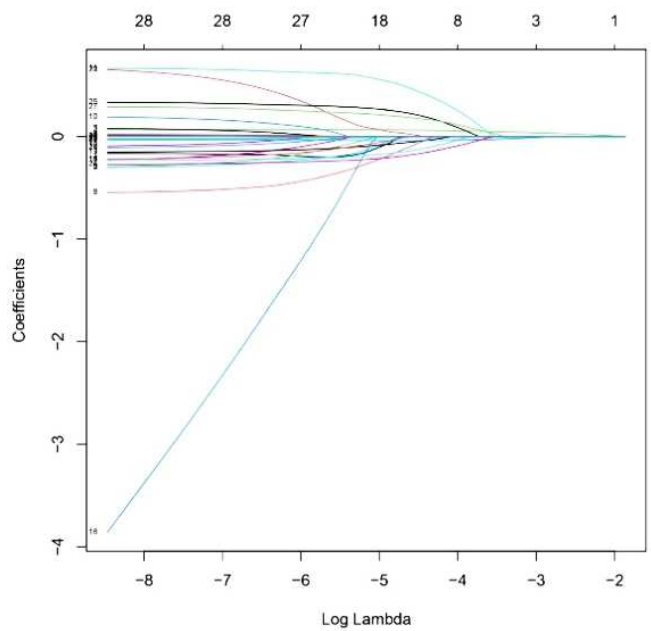

Figure. 1 Perioperative variable selection using a LASSO logistic regression model. A: Dotted vertical lines were depicted at the optimal values by using the minimum criteria (lambda.min) and $1 \mathrm{SE}$ of the minimum criteria (lambda.1se). B: LASSO coefficient profile of 28 variables. The coefficient profile is plotted according to the logarithmic sequence. Five-fold cross-validation via minimum criteria was used to determine the optimal predictors of model resulted in four features with nonzero coefficients.

\section{Construction and validation of the nomogram.}

Multivariable logistic regression analysis based on four predictors screened from LASSO regression analysis was carried out to create the final model (Table 2). The nomogram for predicting $\mathrm{POI}$ in esophageal cancer patients who underwent curative operation was shown in Figure2. Model discrimination, as quantified by the C-index, was $0.802(95 \% \mathrm{Cl}, 0.752-0.852)$, indicating the predictive model can better distinguish $\mathrm{POI}$ patients from non-POI patients (Figure 3A). The calibration plot (Figure 3B) demonstrates good consistency between the predicted risk of $\mathrm{POI}$ and the observed actual risk. The clinical value of the nomograms was assessed by decision curve analysis on the basis of the net benefit and threshold probabilities. As for POI of esophageal cancer, the graph (Figure 3C) suggested the nomogram had superior net benefit with a wide range of threshold probabilities (4-81\%). To confirm the stability of the model, we externally validate the nomogram generated in the training cohort applied to the validation cohort, which composed of 191 esophagectomy patients from August 2011 to December 2011. The predictive nomogram for assessed individual risk of POI, when applied to the validation cohort with a C-index of $0.763(95 \% \mathrm{Cl}, 0.669-0.857)$.

\begin{tabular}{lclc}
\hline Intercept and variable & $\beta$ & Odds ratio $(95 \% \mathrm{Cl})$ & P-value \\
\hline Intercept & -1.91 & $0.15(0.012-2.017)$ & 0.148 \\
Length of stay & 0.07 & $1.07(1.046-1.101)$ & $<0.001$ \\
Albumin & -0.06 & $0.94(0.879-1.004)$ & 0.085 \\
Intraoperative bleeding & 0.00 & $1.00(1.001-1.005)$ & 0.004 \\
Perioperative blood transfusion & 0.28 & $1.32(0.730-2.438)$ & 0.360 \\
\hline
\end{tabular}

Table 2. Prediction factors for the risk of postoperative pulmonary infection with esophageal resection. $\beta$ : the regression coefficient, $\mathrm{Cl}$ : confidence interval. 


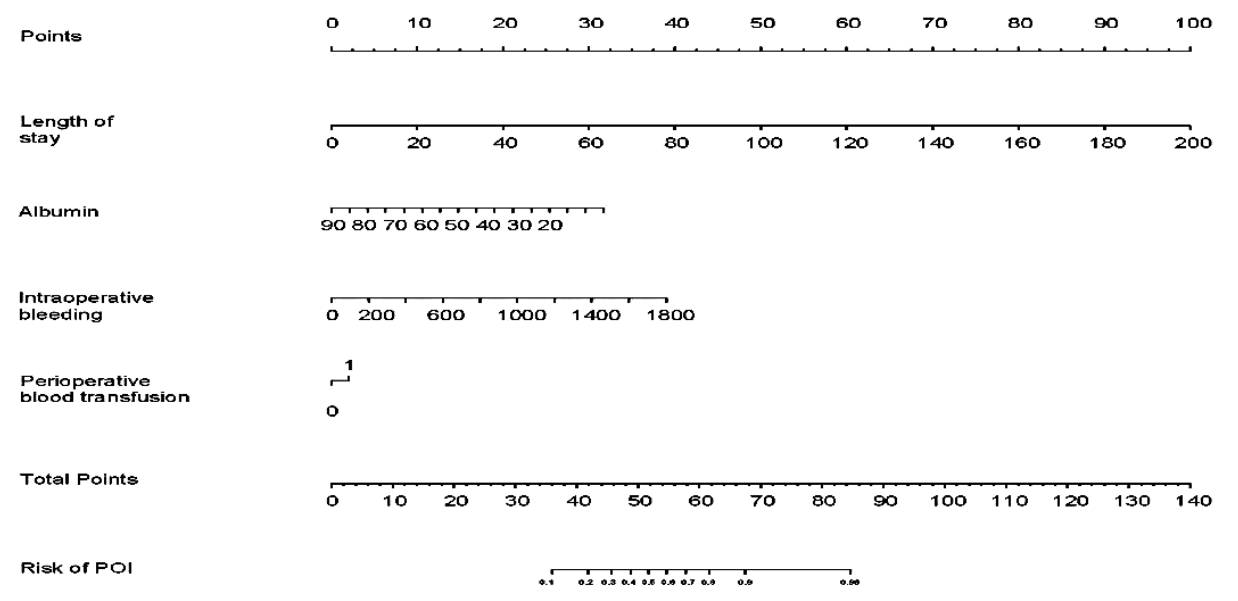

Figure. 2 Nomogram for prediction of $\mathrm{POI}$ in esophageal cancer patients underwent curative operation. POI: postoperative pulmonary infection.

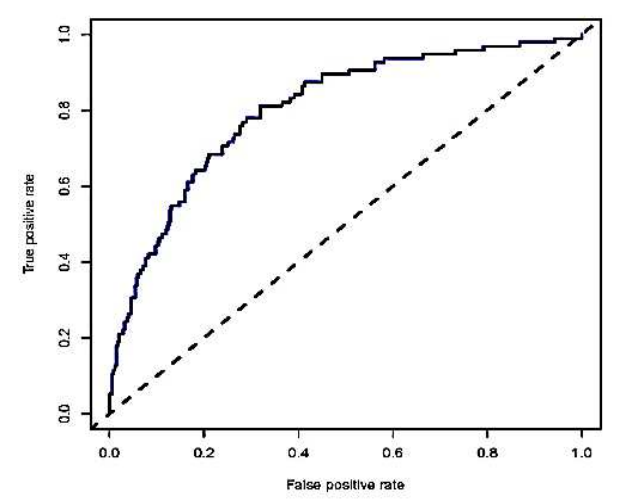

C

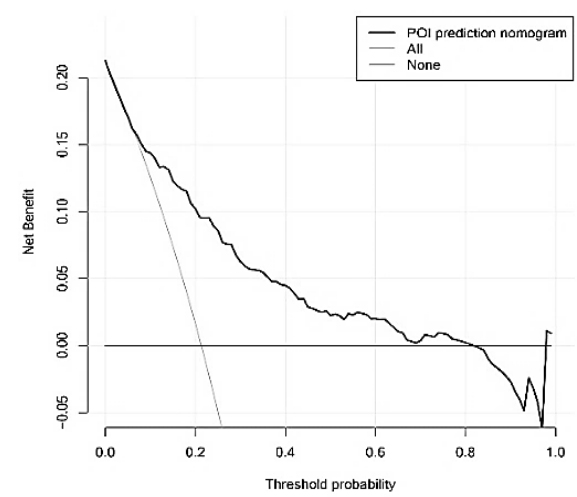

B

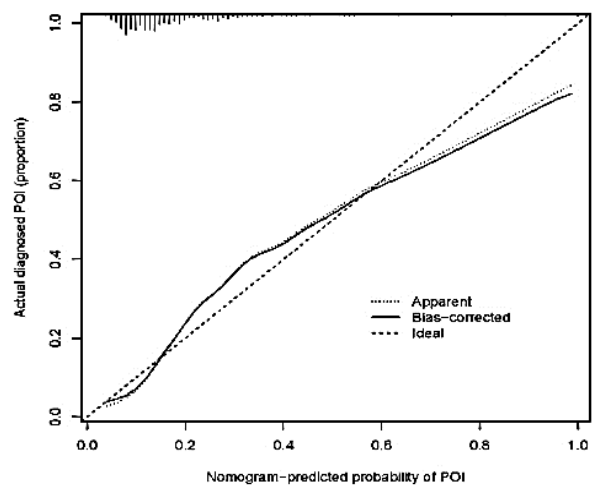

Figure. 3 Evaluation of the nomogram for prediction of $\mathrm{POI}$ in esophageal cancer patients who underwent

esophagectomy.

A: Receiver-operating characteristic curve (ROC) of nomograms for prediction of $\mathrm{POI}$ in esophageal cancer patients. The area under the receiver operating characteristic curve (AUC) value was $0.802(95 \% \mathrm{Cl}, 0.752-0.852)$.

B: Calibration curves of the $\mathrm{POI}$ predictive nomogram in the training cohort.

C: Decision curve analysis for assessing the clinical usefulness of the POI nomogram. In this analysis, the decision curve provided a larger net benefit across the range of 4 and $81 \%$. 


\section{Discussions}

In this study, the incidence of $\mathrm{POI}$ was $20.6 \%$, which is comparable to previous findings $[17,18]$. The multivariable regression analysis of this retrospective study showed that length of stay, albumin, intraoperative bleeding, and perioperative blood transfusion were independent factors of POI. Identification of destructive and protective factors as well as perioperative management with care delivery can facilitate the control of $\mathrm{POI}$, which furthers clinical prognosis and overall survival $[17,19]$.

The current study found that length of stay (LOS) accounts for the increasing incidence of POI. This result is in accordance with earlier observation, which showed that extra length of stay attributable to the possibility of developing infection [20]. Prior researchers examined the relationship between LOS and healthcare-associated infection, they found that 1,039 samples of 51,691 patients experienced respiratory infection. A possible explanation for these results is likely to be related to patients with prolonged LOS may undergo bacterial colonization [21].

Our study found that albumin was clinically relevant to the occurrence of respiratory infection. Consistent with the current result, previous study has demonstrated that the rate of respiratory infection occurred in patients with hypoalbuminemia after radical esophagectomy obviously increased [22]. This result may be explained by the fact that hypoalbuminemia contributes to the decrease of plasma osmotic pressure, which induces pulmonary interstitial edema. In addition, reduced gas dispersion and abnormal ventilation to blood flow ratio make pulmonary infections appeal to occur in patients with esophageal cancer. On the other hand, hypoalbuminemia patients were exposed to pulmonary infection by impairing the immunity of patients, which was frequently observed in patients who underwent resection. Moreover, previous researches have noted that hypoalbuminemia played a great role in the prediction value for the mortality and morbidity rates in esophageal cancer population [23].

The multivariate regression analysis revealed that respiratory infection after esophagectomy could be attributed to intraoperative bleeding. With respect to respiratory infection after curative esophagus surgery, several researchers observed that higher blood loss was identified as an independent risk factor for pulmonary infection [19,24]. Furthermore, for patients with respiratory infection after esophagectomy, greater blood loss was an important indicator of mortality. Increased intraoperative blood loss has been shown to be associated with an increasing incidence of pulmonary complications and hospital death after esophagectomy [25].

In this study, perioperative blood transfusion was found to responsible for POI. There are similarities between the attitudes expressed by researchers in prior studies, they all agreed on the correlation between blood transfusion and increasing susceptibility to pulmonary infection $[26,27]$. Perioperative blood transfusion may work collaboratively with operational stress to induce immunosuppression, which was considered as potential mechanisms of increasing in pulmonary infection following esophagectomy [28].

This retrospective study suggested that length of stay, albumin, intraoperative bleeding, and perioperative blood transfusion were independent factors of POI. For example, if a patient was hospitalized for 60 days, had transfused blood, had an albumin level of 25 , and had intraoperative bleeding of $800 \mathrm{ml}$, his total score is approximately 72.5 points corresponded to approximately $93 \%$ risk of $\mathrm{POI}$.

This study is subject to certain limitations. In this retrospective study, the type of specificinfected bacteria could not be certain. Moreover, the additional disadvantage of this study was 
the limited sample of participants. Additionally, the study is limited by the lack of information on sufficient variables. Some potential variables are not accessible in the database, such as patient-related factors (economic status, social support, education level, health knowledge) and perioperative factors (anesthesia method, Intubation method, medication status, other complications).

\section{Conclusion}

This research identified that length of stay, albumin, intraoperative bleeding, and perioperative blood transfusion emerged as reliable predictors of POI. The finding has important implications for paying much attention to EC patients with postoperative infection and the externally validated nomogram can help prevent the occurrence of postoperative infection cases. Further studies, which take other clinically-relevant variables into account, will perfect the nomogram.

\section{Data availability}

The data contained in this study are available from the corresponding author on reasonable request.

\section{References}

1. Bray, F. et al. Global Cancer Statistics 2018: GLOBOCAN Estimates of Incidence and Mortality Worldwide for 36 Cancers in 185 Countries. CA Cancer J Clin. 68, 394-424 (2018).

2. Hur, Chin et al. Trends in esophageal adenocarcinoma incidence and mortality. Cancer . 119, 1149-58 (2013).

3. Pennathur, Arjun et al. Oesophageal carcinoma. Lancet. 381, 400-12(2013).

4. Chevallay, Mickael et al. Esophageal cancer surgery: review of complications and their management. Ann N Y Acad Sci. 1482, 146-162(2020).

5. Weijs, Teus J et al. Internal and External Validation of a multivariable Model to Define Hospital-Acquired Pneumonia After Esophagectomy. J Gastrointest Surg. 20, 680-7 (2016).

6. van der Sluis, Pieter $C$ et al. A new clinical scoring system to define pneumonia following esophagectomy for cancer. Dig Surg. 31, 108-16(2014).

7. Ohi, Masaki et al. Risk factors and measures of pulmonary complications after thoracoscopic esophagectomy for esophageal cancer. Surg Today. 49, 176-186 (2019).

8. Soutome, Sakiko et al. Prevention of postoperative pneumonia by perioperative oral care in patients with esophageal cancer undergoing surgery: a multicenter retrospective study of 775 patients. Support Care Cancer. 28, 4155-4162 (2020).

9. Dhungel, Birat et al. Patient and peri-operative predictors of morbidity and mortality after esophagectomy: American College of Surgeons National Surgical Quality Improvement Program (ACS-NSQIP), 2005-2008. J Gastrointest Surg. 14, 1492-501 (2010). 10.Zingg, Urs et al. Factors associated with postoperative pulmonary morbidity after esophagectomy for cancer. Ann Surg Oncol. 18, 1460-8 (2011).

11. Grimes, David A. The nomogram epidemic: resurgence of a medical relic. Ann Intern Med. 149, 273-5 (2008).

12. L Levy, Dylan A et al. Development and Validation of Nomograms for Predicting Delayed Postoperative Radiotherapy Initiation in Head and Neck Squamous Cell Carcinoma. JAMA Otolaryngol Head Neck Surg. 146, 455-464 (2020). 
13. Horan, Teresa $C$ et al. CDC/NHSN surveillance definition of health care-associated infection and criteria for specific types of infections in the acute care setting. Am J Infect Control. 36, 309-32 (2008).

14. Rice, Thomas W et al. 7th edition of the AJCC Cancer Staging Manual: esophagus and esophagogastric junction. Ann Surg Oncol. 17, 1721-4 (2010).

15. Liu, Jin et al. Incorporating group correlations in genome-wide association studies using smoothed group Lasso. Biostatistics. 14, 205-19 (2013).

16. Hijazi, Ziad et al. The novel biomarker-based ABC (age, biomarkers, clinical history)bleeding risk score for patients with atrial fibrillation: a derivation and validation study. Lancet. 387, 2302-2311 (2016).

17. Nakamura, Masaki et al. An analysis of the factors contributing to a reduction in the incidence of pulmonary complications following an esophagectomy for esophageal cancer. Langenbecks Arch Surg. 393, 127-33 (2008).

18. Saunders, J H et al. Impact of postoperative complications on disease recurrence and long-term survival following oesophagogastric cancer resection. Br J Surg. 107, 103-112 (2020).

19. Yoshida, Naoya et al. Risk factors for pulmonary complications after esophagectomy for esophageal cancer. Surg Today. 44, 526-32 (2014).

20. Zhou, Qian et al. Estimating extra length of stay and risk factors of mortality attributable to healthcare-associated infection at a Chinese university hospital: a multi-state model. BMC Infect Dis. 19, 975(2019).

21. Darling, Jeremy $D$ et al. Results for primary bypass versus primary angioplasty/stent for lower extremity chronic limb-threatening ischemia. J Vasc Surg. 66, 466-475 (2017).

22. Haridas, Manjunath, and Mark A Malangoni. Predictive factors for surgical site infection in general surgery. Surgery. 144, 496-501 (2008).

23. Kokudo, Takashi et al. Risk factors for incisional and organ space surgical site infections after liver resection are different. World J Surg. 39, 1185-92 (2015).

24. Fang, Wentao et al. Analysis of pulmonary complications after three-field lymph node dissection for esophageal cancer. Ann Thorac Surg. 76, 903-8 (2003).

25. Law, Simon et al. Predictive factors for postoperative pulmonary complications and mortality after esophagectomy for cancer. Ann Surg. 240, 791-800 (2004).

26. Miki, Yuichiro et al. Risk factors for postoperative pneumonia after gastrectomy for gastric cancer. Surg Today. 46, 552-6 (2016).

27. Takeuchi, Daisuke et al. Postoperative complications in elderly patients with gastric cancer. J Surg Res. 198, 317-26 (2015).

28. Xiao, Haifan et al. Development and validation of a prognostic nomogram for predicting post-operative pulmonary infection in gastric cancer patients following radical gastrectomy. Sci Rep. 9, 14587(2019).

\section{Acknowledgement}

This study was funded by the Natural Science Foundation of Shandong Province [grant numbers ZR2015HM066].

\section{Author Contributions}


Gongchao Wang and Shuang Li conceived and designed the study. Shuang Li, Jingwen Su, and Qiyu Sui collected and analyzed the data. Shuang Li wrote and revised the paper. All authors guaranteed that this article has not been published elsewhere.

\section{Additional Information}

Competing Interests: The authors declare no competing interests.

\section{Figure legends}

Figure. 1 Perioperative variable selection using a LASSO logistic regression model. A: Dotted vertical lines were depicted at the optimal values by using the minimum criteria (lambda.min) and 1 SE of the minimum criteria (lambda.1se). B: LASSO coefficient profile of 28 variables. The coefficient profile is plotted according to the logarithmic sequence. Five-fold crossvalidation via minimum criteria was used to determine the optimal predictors of model resulted in four features with nonzero coefficients.

Figure. 2 Nomogram for prediction of $\mathrm{POI}$ in esophageal cancer patients underwent curative operation. POI: postoperative pulmonary infection.

Figure. 3 Evaluation of the nomogram for prediction of $\mathrm{POI}$ in esophageal cancer patients who underwent esophagectomy. A: Receiver-operating characteristic curve (ROC) of nomograms for prediction of $\mathrm{POI}$ in esophageal cancer patients. The area under the receiver operating characteristic curve (AUC) value was $0.802(95 \% \mathrm{Cl}, 0.752-0.852)$. B: Calibration curves of the $\mathrm{POI}$ predictive nomogram in the training cohort. C: Decision curve analysis for assessing the clinical usefulness of the $\mathrm{POI}$ nomogram. In this analysis, the decision curve provided a larger net benefit across the range of 4 and $81 \%$. 


\section{Figures}

A

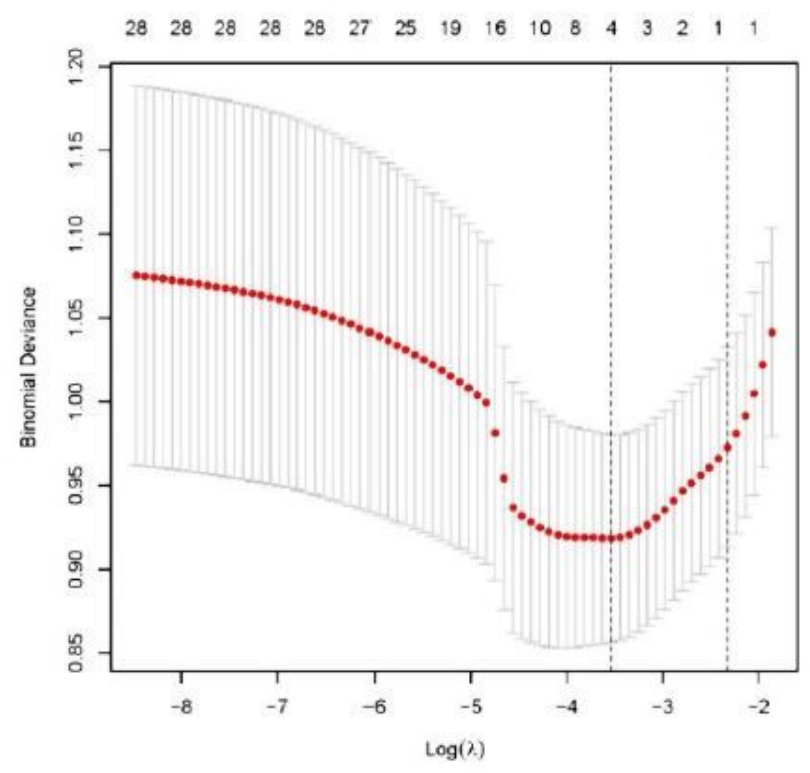

B

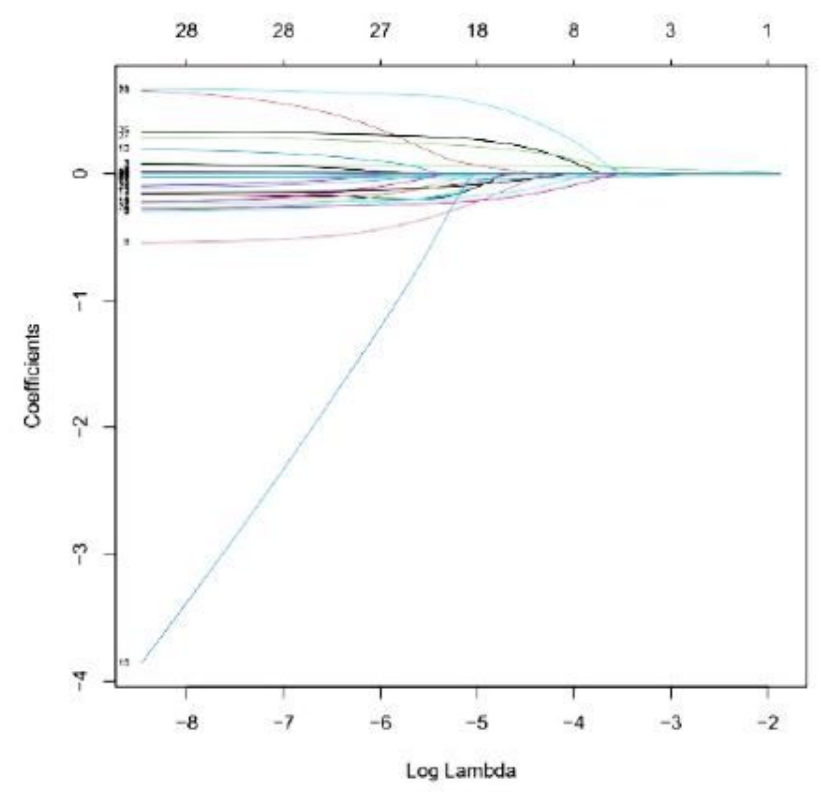

Figure 1

Perioperative variable selection using a LASSO logistic regression model. A: Dotted vertical lines were depicted at the optimal values by using the minimum criteria (lambda.min) and 1 SE of the minimum criteria (lambda.1se). B: LASSO coefficient profile of 28 variables. The coefficient profile is plotted according to the logarithmic sequence. Five-fold cross-validation via minimum criteria was used to determine the optimal predictors of model resulted in four features with nonzero coefficients. 
Points

Length of

stay

Albumin

Intraoperative

bleeding

Periaperative

blood transfusion

Total Points

Risk of $\mathrm{POI}$

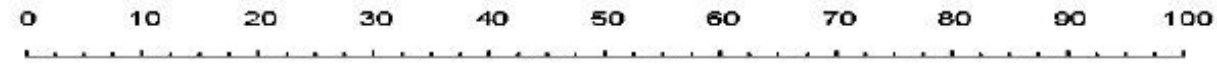

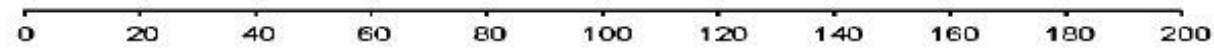

9080706050403020

o $2000^{\prime} 60^{\prime} 1000 ' 1400 ' 1800$

1

0

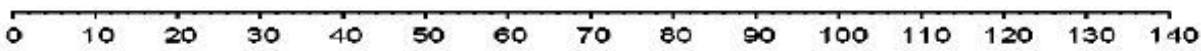

\section{Figure 2}

Nomogram for prediction of POI in esophageal cancer patients underwent curative operation. POI: postoperative pulmonary infection. 
A

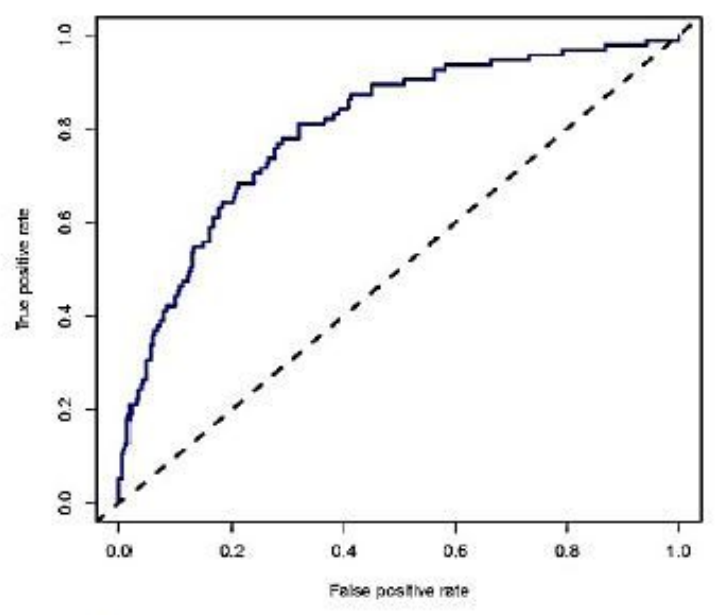

$\mathrm{C}$

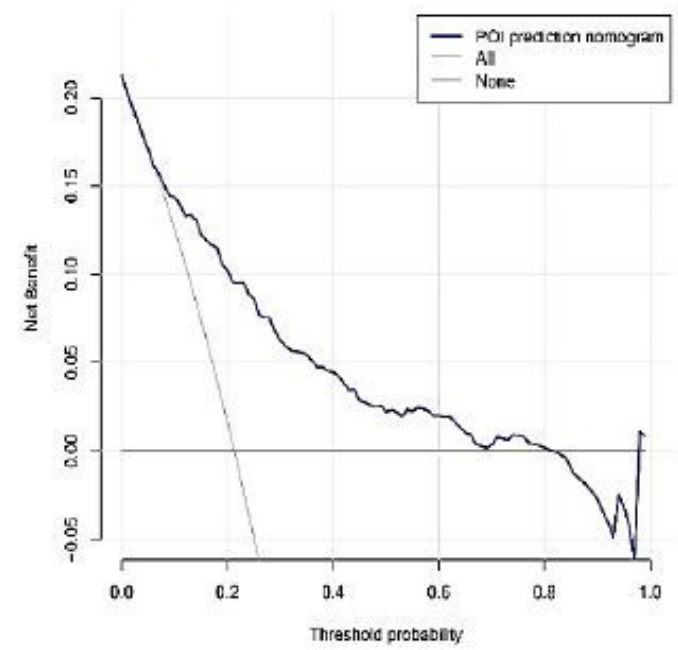

B

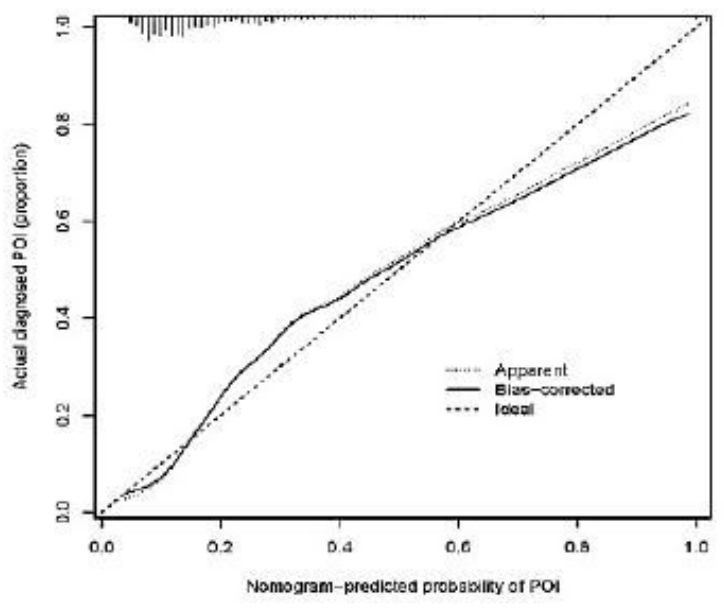

Figure 3

Evaluation of the nomogram for prediction of $\mathrm{POI}$ in esophageal cancer patients who underwent esophagectomy. A: Receiver-operating characteristic curve (ROC) of nomograms for prediction of POI in esophageal cancer patients. The area under the receiver operating characteristic curve (AUC) value was $0.802(95 \% \mathrm{Cl}, 0.752-0.852)$. B: Calibration curves of the POI predictive nomogram in the training cohort. C: Decision curve analysis for assessing the clinical usefulness of the POI nomogram. In this analysis, the decision curve provided a larger net benefit across the range of 4 and $81 \%$. 